\author{
Harnessing Census \\ microdata
}

\section{Social and cultural influences in the digital age}

\section{Continued value of \\ Census data}

with the 2021 Census was great news, however, there will also be new open data sources that will help to change and enhance it.

Barry Leventhal from BarryAnalytics introduced the concept of microdata - samples of raw data on households and individuals drawn from the Census - and summarized its history in the United Kingdom. Following the 2011 Census, microdata products have been produced at three levels of access: public use, safeguarded and secure. For business users, the safeguarded product is likely to be of greatest interest; however, access may be more difficult. Leventhal presented case study examples of applying microdata and summarized the main use cases in market research.

Richard Webber of OriginsInfo discussed the importance of neighbourhoods and how much information can be abstracted about people from the neighbourhoods in which they live. Webber demonstrated the importance of neighbourhood effect in various situations, including performance of schoolchildren and voting behaviour. While digital data can deliver highly specific, up-to-date details about consumers, neighbourhood data can supply contextual effects. He provided a number of examples of 'context' and concluded that digital and contextual sources should be used in combination, as both contribute towards consumer decisions.

\section{Summary}

In summary, this was a fascinating, multifaceted day with a particular focus on open data. The key messages that came through were on the great potential of open sources - data inputs are best used in combination with one another and are best selected on a 'fit-for-purpose' basis. Finally, the Census continues to provide a number of unique views of the population that are unavailable at present from any other sources, and neighbourhood contextual effects remain important - even in the current era of big data.

An earlier version of this review exists as a report on the Harnessing Open Data for Business Advantage seminar and can be accessed here: http://www.mrs.org.uk/page/8448.

Barry Leventhal

\section{IDM Data Council discussion - Consent and convenience in data collection}

Journal of Direct, Data and Digital Marketing Practice (2015) 16, 238-240. doi:10.1057/dddmp.2015.17

At the most recent session of the IDM Data Council on 4 February, there was an open discussion around the nature of the value exchange between 


\section{Google's privacy policy challenged}

\author{
Facebook under \\ investigation
}

\section{Is consumer consent informed?}

\section{Cookies are no barrier}

One in, all in

\section{Risks to the customer experience}

the providers of goods and services and the individuals whose data they seek to capture. In the background of this deliberation was, of course, ongoing consideration by legislators in the European Union of a new Data Protection Regulation that may impose more specific and separate consents for data to be used in order to deliver the service or when it is used for marketing.

But more specific actions by European data protection authorities have foregrounded a gap in current practice before any change in the law. Google signed an undertaking with the Information Commissioner's Office just before the discussion took place that it would amend its privacy policy, following ongoing discussions with regulators in the wake of the attempt by Google to impose a 'one-for-all' opt-in in 2012. While Google is still maintaining its unified policy, it has promised to make access to its privacy policy clearer, easier to find and more comprehensive, and to ensure protection across all devices.

Two days after the council meeting, a group of European data protection authorities from Belgium, The Netherlands, Germany and Italy decided to pursue Facebook over the new privacy policy it had implemented at the end of January. This sees the social network assuming the right to share opted-in data across all of its services, from Instagram to the Atlas ad unit, on the basis of a single permission. The new task force has taken the view that this contravenes European privacy laws.

Tension between the two biggest data controllers on the planet and European regulators and legislators serves to underline just how challenging it now is to ensure that fully compliant, transparent and fair data protection policies are in place. In particular, it is an open question as to whether consumers are giving informed consent, conscious that when they access a service (such as photo blogging or social networks) or download an app, their agreement to its terms and conditions is also agreement to data being used by the provider, its marketing team, associated companies and other partners.

Within the council there were several opposing views on this issue. On one side, it was pointed out that virtually all consumers now consent to cookies, often without thinking about it. Despite the concerns that had driven the change to the ePrivacy Directive in 2011 and led to the requirement to use a cookies notice upfront, most websites now use implied consent (ie, when a visitor progresses past the first screen or dismisses the pop-up) for the opt-in.

It was pointed out that among younger consumers who have only known a world with mobile phones and the internet, there is little fear or concern about data being captured and a greater appetite to get on with an interaction. Peer group adoption of a service or app was identified as a major reason for younger consumers to sign up - if their friends have it, they need it, too. But this has led to some reckless behaviour, such as under-18s engaging in 'sexting', which is illegal at that age.

For the companies represented on the Data Council, the need to capture permission in each channel or when a customer passes from one service to another was thought to be burdensome and a likely barrier to a good 


\section{Data in exchange for services}

\section{The need to monetize data}

\section{How valuable is my data?}

\section{No backlash, only anxiety}

customer experience. But there was also acceptance that permissions have been over-used in the past, such as with email opt-ins, and that a more nuanced approach is likely to be necessary. Applying a one-for-all policy, as Google and Groupon have done, is not only unlikely to comply with European laws, but also risks alienating a customer - if they become disenchanted with one channel or service, they may switch off permission for all of them.

On the other side of the discussion, the view was put forward that this trade-off might be too unconscious among consumers to be realistically thought of as consent. If the details are supplied, but only buried within lengthy terms and conditions, very few individuals will ever read what they are signing up for. At the same time, the providers of these services are not being clear that providing data is the price of entry access is given for free in most cases on the basis that data will be used to target advertising and this is how the service provider funds the business.

It was pointed out that this business model has already given rise to both a degree of consumer concern and alternative marketing by rivals. When it became more widely understood that Google's Gmail service scanned the content of messages in order to deliver contextually relevant advertising, some consumers saw this as an invasion of their privacy. Microsoft picked up on these fears by running a mocking campaign and promoting its privacy-led agenda. A social network aiming to rival Facebook was recently launched that did not carry advertising or harvest its users' data, but demand was so great that it had to close its doors and is currently only available to pre-vetted users. It has also yet to determine how to fund the cost of the service and its associated data centres.

Information exchanges, where consumers actively solicit marketing in exchange for money or discounts, have long been mooted, especially by advocates of personal information management systems. However, the value of an individual's data is often much lower than assumed, it was pointed out, and benefits only tend to accrue in group situations.

Although regulators may be pursuing alleged abuses of trust and permission, the council noted that there was only very limited evidence of a consumer backlash - and then usually only in specific circumstances when an issue was highlighted by the media. For now, the majority are happy to accept the existing value exchange (even if they are not fully aware of it) while companies such as those on the council fret behind the scenes about the sustainability of it all.

David Reed FIDM 